\title{
Risk factors for high-volume lymph node metastases in cNO papillary thyroid microcarcinoma
}

\author{
Chunhao Liu ${ }^{1 \#}$, Yuewu Liu ${ }^{1 \#}$, Lei Zhang', Yunwei Dong ${ }^{3}$, Shenbao Hu${ }^{4}$, Yu Xia ${ }^{5}$, Bo Zhang ${ }^{5}$, Yue Cao ${ }^{1}$, \\ Ziwen Liu ${ }^{1}$, Ge Chen ${ }^{1}$, Zhonghua Shang ${ }^{6}$, Jinbao Yang ${ }^{7}$, Qinghe Sun ${ }^{8}$, Xiaoyi Li $^{1}$ \\ ${ }^{1}$ Department of General Surgery, ${ }^{2}$ Department of Liver Surgery, Peking Union Medical College Hospital, Chinese Academy of Medical Sciences \\ \& Peking Union Medical College, Beijing 100730, China; ${ }^{3}$ Department of General Surgery, Xinzhou People's Hospital, Xinzhou 034000, China; \\ ${ }^{4}$ Department of General Surgery, Jingmen First People's Hospital, Jingmen 448000, China; ${ }^{5}$ Department of Ultrasound, Peking Union Medical \\ College Hospital, Chinese Academy of Medical Sciences \& Peking Union Medical College, Beijing 100730, China; ${ }^{6}$ Department of General Surgery, \\ Second Hospital of Shanxi Medical University, Taiyuan 030001, China; ${ }^{7}$ Department of General Surgery, People's Liberation Army Bethune \\ International Peace Hospital, Shijiazhuang 050082, China; ${ }^{8}$ Department of General Surgery, Cangzhou People's Hospital, Cangzhou 061000, China \\ Contributions: (I) Conception and design: X Li, C Liu; (II) Administrative support: None; (III) Provision of study materials or patients: Y Liu, Y \\ Dong, S Hu, Y Xia, B Zhang, Y Cao, Z Liu, G Chen, Z Shang, J Yang, Q Sun; (IV) Collection and assembly of data: C Liu, L Zhang; (V) Data \\ analysis and interpretation: C Liu, Y Liu, L Zhang; (VI) Manuscript writing: All authors; (VII) Final approval of manuscript: All authors. \\ \#These authors contributed equally to this work. \\ Correspondence to: Xiaoyi Li. General Surgery Department, Peking Union Medical College Hospital, Chinese Academy of Medical Sciences \& Peking \\ Union Medical College, No.1 Shuai Fu Yuan, Dongcheng District, Beijing 100730, China. Email: li.xiaoyi@263.net.
}

Background: Lymph node metastasis (LNM) often occurs in clinical lymph node negative (cN0) papillary thyroid microcarcinoma (PTMC). The risk factors for LNM, especially for high-volume LNM, were investigated in this study.

Methods: The medical records of 1,974 consecutive PTMC patients admitted to the Peking Union Medical College Hospital (PUMCH) from 2013 to 2015 were reviewed. Their clinicopathological features were collected. Univariate and multivariate analyses were performed to identify the risk factors for LNM/high-volume LNM.

Results: Of all the patients, cervical lymph node metastases were detected in 690 patients (34.95\%), and high-volume LNM was detected in 75 patients $(3.80 \%)$. The results of univariate analysis revealed that sex, age, chronic thyroiditis, multifocality, and tumor diameter were significantly correlated with LNM $(\mathrm{P}<0.05)$ and that sex, age, multifocality, and tumor diameter were significantly correlated with high-volume LNM $(\mathrm{P}<0.05)$. Multivariate logistic regression analysis demonstrated that male sex [odds ratio $(\mathrm{OR})$ $=1.657, \mathrm{P}<0.001]$, multifocality $(\mathrm{OR}=1.601, \mathrm{P}<0.001)$, and tumor diameter $>0.5 \mathrm{~cm}(\mathrm{OR}=1.770, \mathrm{P}<0.001)$ were independent risk factors for LNM; age of 40-59 years old ( $\mathrm{OR}=0.427, \mathrm{P}<0.001$ ), age $\geq 60$ years old $(\mathrm{OR}=0.291, \mathrm{P}<0.001)$, and chronic thyroiditis $(\mathrm{OR}=0.562, \mathrm{P}<0.001)$ were independent protective factors for LNM. For high-volume LNM, male sex $(\mathrm{OR}=2.250, \mathrm{P}=0.002)$, tumor diameter $>0.5 \mathrm{~cm}(\mathrm{OR}=3.664$, $\mathrm{P}=0.013)$ and multifocality $(\mathrm{OR}=2.034, \mathrm{P}=0.004)$ were independent risk factors, whereas age $\geq 40$ years old $(\mathrm{OR}=0.240, \mathrm{P}<0.001)$ was an independent protective factor.

Conclusions: Lymph node metastases are common in cN0 PTMC, whereas high-volume LNM is rare. Active surveillance may be reasonable for patients with tumor diameter $\leq 0.5 \mathrm{~cm}$, age $\geq 40$ years old, female sex and isolated lesions.

Keywords: Clinical lymph node negative; high-volume lymph node metastases; papillary thyroid microcarcinoma (PTMC); risk factors

Submitted Jun 16, 2019. Accepted for publication Sep 27, 2019.

doi: 10.21037 /gs.2019.10.04

View this article at: http://dx.doi.org/10.21037/gs.2019.10.04 


\section{Introduction}

Papillary thyroid carcinoma (PTC) is the most common solid tumor of the thyroid gland, accounting for approximately $90 \%$ of all thyroid malignancies (1). In recent years, the incidence of PTC has rapidly increased, and it has become the most common malignant tumor in Korea (2). Of all PTCs, the increase in papillary thyroid microcarcinoma (PTMC) is most pronounced and constitutes half of all new cases. The overall prognosis of these patients is good, and long-term survival rates can reach greater than 99\% (3-5).

Although the survival prognosis of PTMC is good, some patients still face the risk of recurrence during long-term survival. In the literature, the recurrence rates for patients with low-volume and high-volume lymph node metastasis (LNM) are approximately $5 \%$ and $20 \%$, respectively (6). Therefore, the American Thyroid Association (ATA) updated the recurrence risk stratification in 2015 and defined high-volume LNM ( $>5$ involved lymph nodes) as an intermediate risk factor for recurrence (7).

Among PTMCs identified by postoperative pathologic examination, LNM is common, and the proportion of LNM is up to $40 \%$ (8). However, preoperative ultrasound is not sufficiently sensitive to predict LNM in the central compartment of the neck (9). Therefore, a large number of cN0 patients may have LNM $(10,11)$. Prophylactic neck dissection is always controversial in these patients. Those who do not advocate prophylactic neck dissection think that prophylactic neck dissection does not improve prognosis and increases complications (6); however, those who advocate prophylactic neck dissection think that it may alleviate local recurrence and help to assess the risk of recurrence (12-15). Therefore, identifying patients with high-risk LNM among cN0 PTMC patients, especially those with potential high-volume LNM, and providing reasonable treatment have important clinical value. In the past, cN0 PTMC studies focused on the risk factors for LNM, and few studies focused on the rate of and risk factors for high-volume LNM. The preliminary aim of this study is to evaluate the risk factors for $\mathrm{cNO}$ PTMC patients with LNM, and the main aim is to evaluate the risk factors for high-volume LNM by analyzing the clinicopathological data of these patients.

\section{Methods}

\section{Study population}

This historical cohort study included a consecutive series of 1,974 PTMC patients (males: 442, females: 1,532) who underwent primary surgical treatment in the General Surgery Department of Peking Union Medical College Hospital (PUMCH) from November 2013 to October 2015. The study was approved by the Ethics Committees of Peking Union Medical Hospital, and informed consent was obtained from all patients.

In this study, LNM of the neck was evaluated by ultrasonography. If the following signs were not observed, LNM was defined as cN0: cervical lymph node transverse/ long diameter $>0.5$, corticomedullary demarcation was unclear or the medulla structure disappeared, microcalcification or cystic changes similar to the original foci, high echogenic mass within the cortex, abundant blood flow around the cortex or irregular blood flow (16).

Patients were included if they exhibited the following criteria: (I) newly diagnosed PTC of which the largest diameter was $<1 \mathrm{~cm}$; in cases of multifocal PTMCs, the size of the largest tumor was used; (II) met the cN0 diagnostic criteria; and (III) primary surgical treatment based on Chinese guidelines: lobectomy or near-total/total thyroidectomy with neck lymph node dissection (ipsilateral central compartment dissection for patients with malignant lesion(s) in one lobe/bilateral central compartment dissection for patients with bilateral malignant lesions). Patients were excluded based on the following criteria: (I) preoperatively confirmed cN1; (II) visiting for revision surgery; (III) not undergoing cervical lymph node dissection; and (IV) histologically confirmed non-PTC. The medical records of all included patients were reviewed to extract the following items: sex, age, chronic thyroiditis, capsule invasion, multifocality, and tumor diameter. The above items were analyzed to identify the risk factors for LNM/high-volume LNM. Patients were allocated to three groups $(8,17)$ : group A: $0-39$ years old; group B: $40-59$ years old; and group C: $\geq 60$ years old. In multifocal cases, the size of the largest tumor was recorded for data analysis. The extent of central neck lymph node dissection was based on the 2009 ATA guidelines (18). The presence and number of metastatic lymph nodes were determined via surgical pathology. High-volume LNM was defined as $>5$ metastatic lymph nodes.

\section{Statistical analysis}

SPSS statistical software 22.0 (version 22.0; SPSS Inc., Chicago, IL, USA) was used for the statistical analyses. The differences between patients with and without LNM/high- 
Table 1 Univariate analysis of risk factors for LNM and high-volume LNM in cN0 PTMC patients

\begin{tabular}{|c|c|c|c|c|c|c|}
\hline Item & \multicolumn{3}{|c|}{ LNM } & \multicolumn{3}{|c|}{$>5$ LNM } \\
\hline Sex & & & $<0.001$ & & & 0.001 \\
\hline Female & $1,045(68.21)$ & 487 (31.79) & & $1,487(97.06)$ & $45(2.94)$ & \\
\hline Male & $239(54.07)$ & $203(45.93)$ & & $412(93.21)$ & $30(6.79)$ & \\
\hline Group A & 305 (51.09) & $292(48.91)$ & & 548 (91.79) & $49(8.21)$ & \\
\hline Group B & $880(70.46)$ & $369(29.54)$ & & 1,223 (97.92) & $26(2.08)$ & \\
\hline Group C & 99 (77.34) & $29(22.66)$ & & $128(100.00)$ & $0(0.00)$ & \\
\hline Chronic thyroiditis & & & $<0.001$ & & & 0.274 \\
\hline Capsule invasion & & & 0.039 & & & 0.177 \\
\hline$(-)$ & $842(66.72)$ & $420(33.28)$ & & $1,220(96.67)$ & $42(3.33)$ & \\
\hline$(+)$ & $442(62.08)$ & $270(37.92)$ & & $679(95.37)$ & $33(4.63)$ & \\
\hline Multifocality & & & $<0.001$ & & & 0.007 \\
\hline$(-)$ & $919(68.23)$ & $428(31.77)$ & & 1,307 (97.03) & $40(2.97)$ & \\
\hline$(+)$ & $365(58.21)$ & $262(41.79)$ & & $592(94.42)$ & $35(5.58)$ & \\
\hline Tumor diameter ${ }^{\mathrm{a}}(\mathrm{cm})$ & & & $<0.001$ & & & $<0.001$ \\
\hline$\leq 0.5$ & $304(75.81)$ & 97 (24.19) & & $397(99.00)$ & $4(1.00)$ & \\
\hline
\end{tabular}

a , diameter of the largest lesion in multifocal tumors. LNM, lymph node metastases; PTMC, papillary thyroid microcarcinoma.

volume LNM were examined using Fisher's exact test or the $\chi^{2}$ test. Risk factors for LNM/high-volume LNM were identified by univariate analysis and multivariate logistic regression models. Statistical significance was defined as $\mathrm{P}<0.05$.

\section{Results}

Among all the patients, cervical lymph node metastases were detected in 690 patients $(34.95 \%)$, and highvolume LNM was detected in 75 patients $(3.80 \%)$. In the univariate analysis, compared with the parameters in the control group, male sex $(45.93 \%$ vs. $31.79 \%, \mathrm{P}<0.05)$, age $<40$ years old ( $<40$ years old, $48.91 \%$; 40-59 years old, $29.54 \%$; $\geq 60$ years old $22.66 \%, \mathrm{P}<0.05)$, multifocality (41.79\% vs. $31.77 \%, \mathrm{P}<0.05)$, without chronic thyroiditis (38.20\% vs. $25.05 \%, \mathrm{P}<0.05)$, and tumor diameter $>0.5 \mathrm{~cm}$
(37.70\% vs. $24.19 \%, \mathrm{P}<0.05)$ were significantly associated with LNM (Table 1). In the multivariate analysis, compared with young patients (group A), middle-age [odds ratio (OR) $=0.427,95 \%$ confidence interval $(\mathrm{CI}): 0.347-0.526$, group B] and elderly (OR $=0.291,95 \%$ CI: $0.185-0.459$, group C) patients were independent protective factors for LNM. In contrast, male sex (OR $=1.657,95 \%$ CI: 1.319-2.082), multifocality (OR $=1.601,95 \%$ CI: $1.303-1.967)$, and tumor diameter $>0.5 \mathrm{~cm}(\mathrm{OR}=1.770,95 \% \mathrm{CI}$ : $1.362-2.299)$ were independent risk factors for LNM (Table 2).

Furthermore, univariate analysis was performed for 75 patients with high-volume LNM and 1,899 patients without high-volume LNM. Compared with the parameters in the control group, male sex $(6.79 \%$ vs. $2.94 \%, \mathrm{P}<0.05)$, age $<40$ years old (age $<40$ years, $8.21 \%$; age of $40-59$ years old, $2.08 \%$; age $\geq 60$ years old, $0.00 \%, \mathrm{P}<0.05$ ), multifocality $(5.58 \%$ vs. $2.97 \%, \mathrm{P}<0.05)$ and tumor diameter $>0.5 \mathrm{~cm}$ 
Table 2 Multivariate analysis of risk factors for LNM in cN0 PTMC patients

\begin{tabular}{lccc}
\hline Item & OR & $95 \% \mathrm{Cl}$ & - \\
\hline Group A & 1.000 & - & $<$ value \\
Group B & 0.427 & $0.347-0.526$ & $<0.001$ \\
Group C & 0.291 & $0.185-0.459$ & $<0.001$ \\
Male & 1.657 & $1.319-2.082$ & $<0.001$ \\
Multifocality & 1.601 & $1.303-1.967$ & $<0.001$ \\
Tumor diameter $>0.5 \mathrm{~cm}$ & 1.770 & $1.362-2.299$ & $<0.001$ \\
Chronic thyroiditis & 0.562 & $0.441-0.715$ & $<0.001$ \\
\hline
\end{tabular}

OR, odds ratio; Cl, confidence interval; LNM, lymph node metastases; PTMC, papillary thyroid microcarcinoma.

Table 3 Multivariate analysis of risk factors for high-volume LNM in cN0 PTMC patients

\begin{tabular}{lccc}
\hline Item & OR & $95 \% \mathrm{Cl}$ & P value \\
\hline Group A & 1.000 & - & $<$ \\
Group B & 0.240 & $0.146-0.393$ & 0.001 \\
Multifocality & 2.034 & $1.258-3.289$ & 0.004 \\
Male & 2.250 & $1.363-3.714$ & 0.002 \\
Tumor diameter $>0.5 \mathrm{~cm}$ & 3.664 & $1.310-10.250$ & 0.013 \\
\hline
\end{tabular}

OR, odds ratio; $\mathrm{Cl}$, confidence interval; LNM, lymph node metastases; PTMC, papillary thyroid microcarcinoma.

( $4.51 \%$ vs. $1.00 \%, \mathrm{P}<0.05$ ) were significantly associated with high-volume LNM (Table 1). Multivariate analysis revealed that compared with young patients (group A), middleage $(\mathrm{OR}=0.240,95 \%$ CI: 0.146-0.393, group B) was an independent protective factor for high-volume LNM. In contrast, male sex (OR =2.250, 95\% CI: 1.363-3.714), multifocality (OR $=2.034,95 \%$ CI: $1.258-3.289)$, and tumor diameter $>0.5 \mathrm{~cm}(\mathrm{OR}=3.664,95 \% \mathrm{CI}: 1.310-10.250)$ were independent risk factors for high-volume LNM (Table 3).

\section{Discussion}

Neck ultrasound and CT are currently widely used for the screening of metastatic lymph nodes $(19,20)$. Ultrasound offers advantages, including the ease of use and short operation time, and is superior to CT for lymph node microstructure recognition. Thus, ultrasound is recommended as the main technique for preoperative diagnosis. Due to the anatomical features of the neck, the accuracy of ultrasound in the diagnosis of central LNM is not satisfactory. In our previous study, we reported an LNM rate of $9.79 \%(120 / 1,226)$ based on preoperative ultrasonography, and the sensitivity of preoperative ultrasonography was $17.35 \%$ (76/438) for LNM. However, postoperative pathological findings revealed that the LNM rate was $35.73 \%(438 / 1,226)(9,21,22)$. Therefore, a significant proportion of $\mathrm{cN} 0$ patients have confirmed LNM after surgery. In this study, the LNM rate was $34.95 \%$ in patients with cN0 PTMC. Previous studies have demonstrated that male sex, tumor diameter, and age are independent risk factors for LNM in PTMC patients (23). Our study reconfirmed similar results: the LNM rates for age $<40$ years old, male sex, multifocality, and tumor diameter $>0.5 \mathrm{~cm}$ were $48.91 \%, 45.93 \%$, $41.79 \%$, and $37.70 \%$, respectively. These characteristics represent independent risk factors for cN0 PTMC patients with LNM. Therefore, LNM occurs in approximately $1 / 3$ of cN0 PTMC patients. These results suggest that prophylactic central neck dissection has certain rationality for patients with high-risk factors for LNM, and some guidelines have also recommended routine prophylactic central neck dissection $(24,25)$. The tumor stage and treatment strategies for these patients may change (26).

However, it is necessary to carefully consider the clinical 
significance of small metastatic lymph nodes (lesions that cannot be detected by preoperative ultrasonography) and their influence on survival or recurrence prognosis. Studies have demonstrated that the overall prognosis of PTMC is good. The long-term survival rate can reach greater than $99 \%$. The local or regional recurrence rate is $2 \%$ to $6 \%$, and the rate of distant metastases is only $1 \%$ to $2 \%(3-5)$. According to two studies from Korea and Australia, the lymph node dissection rates were $91.3 \%(\mathrm{n}=2,018)$ and $21.7 \%(\mathrm{n}=1,391)$, respectively, and the LNM rates were $34.1 \%$ and $3.2 \%$, respectively. However, the 5- and 7-year recurrence rates were $3.2 \%$ and $0.6 \%$, respectively, in the two studies $(27,28)$. Thus, we can infer that small metastatic lymph nodes (that cannot be detected by preoperative ultrasonography) that may be missed in surgery without prophylactic neck dissection will not progress over a long period of time and have little significance in clinical practice. These metastatic lymph nodes may have limited prognostic impact in PTMC patients (29). In fact, a number of autopsy studies have reported that occult papillary carcinoma exists in $1 \%$ to $35.6 \%$ of "ordinary people" (average $11.5 \%$ ), and $10 \%$ of this population has occult LNM (14). Therefore, PTC and its metastatic lesions may be present in the "normal population", and these lesions may not threaten these individuals throughout their lives. In a study assessing the observation of low-risk PTMC patients who did not undergo immediate surgery, the incidence rates of the novel appearance of lymph-node metastases were $1.7 \%$ and $3.8 \%$ in 1,235 PTMC patients after 5 and 10 years, respectively (17), and these rates are similar to the highvolume LNM rate $(3.80 \%)$ found in our study. Therefore, we may infer that high-volume LNM in cNO PTMC has great clinical significance, and we should give priority and treatment to patients with high-volume LNM. Studies have demonstrated that the recurrence rate of patients with highvolume LNM is significantly increased compared with that of patients without high-volume LNM. The 2015 ATA guidelines also identified high-volume LNM as an important risk factor for recurrence risk stratification (6).

This study confirmed that male sex was an independent risk factor for high-volume LNM in patients with $\mathrm{cN} 0$ PTMC ( $\mathrm{OR}=2.250, \mathrm{P}=0.002)$, and older patients exhibited a reduced risk of high-volume LNM compared with younger patients (40-59 years old, $\mathrm{OR}=0.240, \mathrm{P}<0.001$ ); the high-volume LNM rates in males and patients $<40,40-59$, and $\geq 60$ years old were $6.79 \%, 8.21 \%, 2.08 \%$, and 0 , respectively. These results are similar to our previous research conclusions (8). Notably, age stratification in this study was verified as an effective means to predict the risk of LNM and high-volume LNM in cN0 PTMC patients. The treatment strategy for patients $<40$ years could be more aggressive in consideration of the highest LNM and highvolume LNM risk and the long-life span.

Also, tumor size is another risk factor for LNM and highvolume LNM that should be emphasized. The LNM and high-volume LNM rates of patients with tumor diameters $\leq 0.5 \mathrm{~cm}$ were only $24.2 \%$ and $1.0 \%$, respectively (and in the $>0.5 \mathrm{~cm}$ group were $37.7 \%$ and $4.5 \%$, respectively), and multivariate analysis also revealed that the risks of LNM and high-volume LNM were significantly reduced compared with the $>0.5 \mathrm{~cm}$ tumor group. The prognosis of patients with a tumor diameter $\leq 0.5 \mathrm{~cm}$ is significantly better than that of patients with a tumor $>0.5 \mathrm{~cm}$ after treatment (28). Therefore, it may be reasonable and safe to observe $\leq 0.5 \mathrm{~cm}$ tumors and not to provide immediate surgery (30). Some studies have demonstrated that the total tumor diameter of multifocal lesions is positively correlated with the rate of lymph node metastases $(31,32)$. This finding further suggests a relationship between tumor size and lymph node metastases. In this study, multifocality was also identified as an independent risk factor for LNM and high-volume LNM. Moreover, there are some difficulties in the evaluation of multifocal lesions during follow-up, and the evaluation of unifocal lesions is much easier in terms of observation.

\section{Conclusions}

In summary, lymph node metastases are common in $\mathrm{cN} 0$ PTMC, whereas high-volume LNM is rare. For younger patients, male patients, patients with tumors $>0.5 \mathrm{~cm}$ and patients with multifocal lesions, the risks of LNM/highvolume LNM are significantly increased. Therefore, a deliberate treatment decision should be made after communicating with patients; once surgery is decided, prophylactic neck dissection should be considered. Although some studies indicate that prophylactic neck dissection may increase the risk of complications $(6,33)$, prophylactic neck dissection performed by experienced surgeons does not increase the rate of complications and may also reduce recurrence and avoid reoperation (34-37). However, active surveillance may be more reasonable for patients with a tumor diameter $\leq 0.5 \mathrm{~cm}$, age $\geq 40$ years old, female sex and isolated lesions. 


\section{Acknowledgments}

We thank all the patients who participated in this study.

\section{Footnote}

Conflicts of Interest: The authors have no conflicts of interest to declare.

Ethical Statement: The authors are accountable for all aspects of the work in ensuring that questions related to the accuracy or integrity of any part of the work are appropriately investigated and resolved. The study was approved by the Ethics Committees of Peking Union Medical Hospital (No. S-K889), and informed consent was obtained from all patients.

\section{References}

1. Davies L, Welch HG. Current thyroid cancer trends in the United States. JAMA Otolaryngol Head Neck Surg 2014;140:317-22.

2. Jung KW, Park S, Kong HJ, et al. Cancer statistics in Korea: incidence, mortality, survival, and prevalence in 2008. Cancer Res Treat 2011;43:1-11.

3. Hay ID. Management of patients with low-risk papillary thyroid carcinoma. Endocr Pract 2007;13:521-33.

4. Mazzaferri EL. Management of low-risk differentiated thyroid cancer. Endocr Pract 2007;13:498-512.

5. Londero SC, Krogdahl A, Bastholt L, et al. Papillary thyroid carcinoma in Denmark, 1996-2008: outcome and evaluation of established prognostic scoring systems in a prospective national cohort. Thyroid 2015;25:78-84.

6. Haugen BR, Alexander EK, Bible KC, et al 2015 American Thyroid Association Management Guidelines for Adult Patients with Thyroid Nodules and Differentiated Thyroid Cancer: The American Thyroid Association Guidelines Task Force on Thyroid Nodules and Differentiated Thyroid Cancer. Thyroid 2016;26:1-133.

7. Adam MA, Pura J, Goffredo P, et al. Presence and Number of Lymph Node Metastases are Associated with Compromised Survival for Patients Younger Than Age 45 Years With Papillary Thyroid Cancer. J Clin Oncol 2015;33:2370-5.

8. Zhang L, Yang J, Sun Q, et al. Risk factors for lymph node metastasis in papillary thyroid microcarcinoma: Older patients with fewer lymph node metastases. Eur J Surg Oncol 2016;42:1478-82.

9. Zhang L, Liu H, Xie Y, et al. Risk factors and indication for dissection of right paraesophageal lymph node metastasis in papillary thyroid carcinoma. Eur J Surg Oncol 2016;42:81-6.

10. Koo BS, Choi EC, Yoon YH, et al. Predictive factors for ipsilateral or contralateral central lymph node metastasis in unilateral papillary thyroid carcinoma. Ann Surg 2009;249:840-4.

11. Sugitani I, Fujimoto Y, Yamada K, et al. Prospective outcomes of selective lymph node dissection for papillary thyroid carcinoma based on preoperative ultrasonography. World J Surg 2008;32:2494-502.

12. Hartl DM, Mamelle E, Borget I, et al. Influence of prophylactic neck dissection on rate of retreatment for papillary thyroid carcinoma. World J Surg 2013;37:1951-8.

13. Popadich A, Levin O, Lee JC, et al. A multicenter cohort study of total thyroidectomy and routine central lymph node dissection for $\mathrm{cN} 0$ papillary thyroid cancer. Surgery 2011;150:1048-57.

14. Barczyński M, Konturek A, Stopa M, et al. Prophylactic central neck dissection for papillary thyroid cancer. Br J Surg 2013;100:410-8.

15. Lee YC, Na SY, Park GC, et al. Occult lymph node metastasis and risk of regional recurrence in papillary thyroid cancer after bilateral prophylactic central neck dissection: A multi-institutional study. Surgery 2017;161:465-71.

16. Podnos YD, Smith D, Wagman LD, et al. The implication of lymph node metastasis on survival in patients with welldifferentiated thyroid cancer. Am Surg 2005;71:731-4.

17. Ito Y, Miyauchi A, Kihara M, et al. Patient age is significantly related to the progression of papillary microcarcinoma of the thyroid under observation. Thyroid 2014;24:27-34.

18. American Thyroid Association Surgery Working Group; American Association of Endocrine Surgeons; American Academy of Otolaryngology-Head and Neck Surgery, et al. Consensus statement on the terminology and classification of central neck dissection for thyroid cancer. Thyroid 2009;19:1153-8.

19. Kouvaraki MA, Shapiro SE, Fornage BD, et al. Role of preoperative ultrasonography in the surgical management of patients with thyroid cancer. Surgery 2003;134:946-54; discussion 954-5.

20. Som PM, Brandwein M, Lidov M, et al. The varied presentations of papillary thyroid carcinoma cervical nodal disease: CT and MR findings. AJNR Am J Neuroradiol. 1994;15:1123-8. 
21. Choi JS, Kim J, Kwak JY, et al. Preoperative staging of papillary thyroid carcinoma: comparison of ultrasound imaging and CT. AJR Am J Roentgenol 2009;193:871-8.

22. Hwang HS, Orloff LA. Efficacy of preoperative neck ultrasound in the detection of cervical lymph node metastasis from thyroid cancer. Laryngoscope 2011;121:487-91.

23. Sun W, Lan X, Zhang H, et al. Risk Factors for Central Lymph Node Metastasis in CN0 Papillary Thyroid Carcinoma: A Systematic Review and Meta-Analysis. PLoS One 2015;10:e0139021.

24. Takami H, Ito Y, Noguchi H, et al. Treatment of thyroid tumor: Japanese Clinical Guidelines. 2010.

25. Endocrine Society of Chinese Medical Association. Management guidelines of thyroid nodules and differentiated thyroid cancer. Chin J Endocrinol Metab 2012;28:779-97.

26. Pacini F, Castagna MG, Brilli L, et al. Thyroid cancer: ESMO Clinical Practice Guidelines for diagnosis, treatment and follow-up. Ann Oncol 2010;21 Suppl 5:v214-9.

27. Gschwandtner E, Klatte T, Swietek N, et al. Increase of papillary thyroid microcarcinoma and a plea for restrictive treatment: A retrospective study of 1,391 prospective documented patients. Surgery 2016;159:503-11.

28. Lee J, Song Y, Soh EY. Central lymph node metastasis is an important prognostic factor in patients with papillary thyroid microcarcinoma. J Korean Med Sci 2014;29:48-52.

29. Randolph GW, Duh QY, Heller KS, et al. The prognostic significance of nodal metastases from papillary thyroid carcinoma can be stratified based on the size and number of metastatic lymph nodes, as well as the presence of extranodal extension. Thyroid 2012;22:1144-52.

Cite this article as: Liu C, Liu Y, Zhang L, Dong Y, Hu S, Xia Y, Zhang B, Cao Y, Liu Z, Chen G, Shang Z, Yang J, Sun Q, Li $\mathrm{X}$. Risk factors for high-volume lymph node metastases in cN0 papillary thyroid microcarcinoma. Gland Surg 2019;8(5):550-556. doi: 10.21037 /gs.2019.10.04
30. Gao M, Ge MH, Guan HX, et al. Chinese expert consensus on diagnosis and treatment of papillary thyroid microcarcinoma. Chin J Clin Oncol 2016:43:405-11.

31. Tam AA, Ozdemir D, Cuhaci N, et al. Association of multifocality, tumor number, and total tumor diameter with clinicopathological features in papillary thyroid cancer. Endocrine 2016;53:774-83.

32. Al Afif A, Williams BA, Rigby MH, et al. Multifocal Papillary Thyroid Cancer Increases the Risk of Central Lymph Node Metastasis. Thyroid 2015;25:1008-12.

33. Nixon IJ, Wang LY, Ganly I, et al. Outcomes for patients with papillary thyroid cancer who do not undergo prophylactic central neck dissection. Br J Surg 2016;103:218-25.

34. Bonnet S, Hartl D, Leboulleux S, et al. Prophylactic lymph node dissection for papillary thyroid cancer less than $2 \mathrm{~cm}$ : implications for radioiodine treatment. J Clin Endocrinol Metab 2009;94:1162-7.

35. Chisholm EJ, Kulinskaya E, Tolley NS. Systematic review and meta-analysis of the adverse effects of thyroidectomy combined with central neck dissection as compared with thyroidectomy alone. Laryngoscope 2009;119:1135-9.

36. Sancho JJ, Lennard TW, Paunovic I, et al. Prophylactic central neck disection in papillary thyroid cancer: a consensus report of the European Society of Endocrine Surgeons (ESES). Langenbecks Arch Surg 2014;399:155-63.

37. Zhao W, You L, Hou X, et al. The Effect of Prophylactic Central Neck Dissection on Locoregional Recurrence in Papillary Thyroid Cancer After Total Thyroidectomy: A Systematic Review and Meta-Analysis: pCND for the Locoregional Recurrence of Papillary Thyroid Cancer. Ann Surg Oncol 2017;24:2189-98. 